\title{
Prise en charge spécialisée des sarcomes au CHUV
}

\author{
Francesca Bosisio ${ }^{a}$, Maurice Matterb, Aurélie Fortinc et I'Equipe interdisciplinaire sarcomes du CHUV \\ ${ }^{a} \mathrm{PhD}, \mathrm{MSc}$, Direction médicale; ${ }^{\mathrm{b}} \mathrm{MD}, \mathrm{PD}$, Service de chirurgie viscérale; ${ }^{c} \mathrm{MSc}$, Unité d'oncologie interdisciplinaire et réseaux
}

\section{Introduction}

En Suisse, environ 350 cas annuels de sarcomes ont été documentés entre 2009 et 2013 par la Ligue suisse contre le cancer [1] ${ }^{1}$. En 2015, le CHUV a pris en charge 80 patients, dont 9 étaient des enfants. Compte tenu du fait que les sarcomes sont des tumeurs rares, agressives et extrêmement hétérogènes, il est actuellement admis que le diagnostic et le pronostic est meilleur lorsque les patients sont pris précocement en charge dans un centre spécialisé [2-5].

La prise en charge interdisciplinaire des sarcomes au CHUV est un standard depuis plus de vingt ans. En 2013, la décision de formaliser un centre a été motivée par les raisons suivantes:

- La Conférence suisse des directrices et directeurs cantonaux de la santé a intégré la prise en charge des sarcomes pédiatriques aux disciplines de la Médecine hautement spécialisée. Leur prise en charge a été attribuée à quatre hôpitaux, dont le CHUV.

- Les médecins et chercheurs suisses concernés par la prise en charge des sarcomes ont fondé en 2013 SwissSARCOS et le Swiss Sarcoma Advisory Board. Ce groupe a depuis créé un registre national des sar-

accessibles dans l'édition actuelle du BMS: https:// bullmed.ch/\#toc-bar comes et a élaboré des recommandations nationales de prise en charge clinique.

- L'Office fédéral de la santé publique a inauguré un programme national visant l'amélioration de la prise en charge des maladies rares de l'enfant à l'adulte.

La mise sur pied du Centre des sarcomes au CHUV fait suite au Centre du sein [6,7], au Centre de la prostate $[8,9]$ et au Centre des tumeurs thoraciques. En matière de sarcomes, une attention particulière a été prêtée à 1) l'amélioration de la coordination de la prise en charge au sein du CHUV et à 2) encourager les médecins de famille et les spécialistes à référer rapidement leurs patients à un centre spécialisé.

Dans le cadre de cet article, nous décrirons les outils qui nous ont permis d'atteindre ces objectifs. Dans la littérature scientifique anglophone et francophone, les mots «multidisciplinaire», «interdisciplinaire» et "pluridisciplinaire» sont présents. Dans cet article, nous privilégierons le mot «interdisciplinaire» de manière cohérente avec la terminologie utilisée par la Direction médicale et le Département d'oncologie du CHUV.

\section{Amélioration de la coordination de la prise en charge au sein du CHUV}

L'atteinte de cet objectif repose sur les éléments suivants:

- Optimisation du colloque interdisciplinaire des sarcomes

- Adaptation des recommandations internationales de pratique clinique

- Elaboration d'itinéraires cliniques

- Création d'une base de données de centre

\section{Optimisation du colloque interdisciplinaire des sarcomes}

Les colloques sont la pierre angulaire de la prise en charge interdisciplinaire de tumeurs $[10,11]$. Ils permettent en effet aux professionnels de se rencontrer et discuter le diagnostic et le traitement des patients. Comme la littérature met en évidence que l'organisation du colloque interdisciplinaire a une influence sur 
la prise en charge du patient [10,12], la première étape du projet de Centre des sarcomes a été de mieux structurer les colloques - pédiatrique et adulte - existants:

- Identifier les participants obligatoires (chirurgiens, oncologues, radio-oncologues, radiologues, pathologues et infirmières cliniciennes spécialisées) et optionnels (psychologues, assistants sociaux, nutritionnistes, physio- et ergothérapeutes...) et monitorer leur présence. Suivant les recommandations de l'American College of Surgeons, les participants obligatoires doivent prendre part à $75 \%$ des colloques interdisciplinaires [13]. Au Centre des sarcomes, lorsque les participants obligatoires sont absents et ne sont pas remplaçables par leurs remplaçants, la réunion est reportée. Ceci est décidé une semaine à l'avance. En 2015, un seul colloque a été annulé pour cette raison, nous considérons donc cet objectif atteint.

- Les participants optionnels sont encouragés à participer au besoin dans la mesure où leur présence peut améliorer la prise de décision et les recommandations de traitement [14].

- Rendre obligatoire la présentation pré- et postthérapeutique de chaque patient. Les critères des Centres du sein [7] et de la prostate [9] recommande aussi qu'au moins 90\% des patients soient discuté dans le cadre d'un colloque interdisciplinaire. $\mathrm{Au}$ Centre des sarcomes, 98\% des patients ont été discutés en 2015 et les patients sont présentés en moyenne trois fois.

- Planifier des colloques interdisciplinaires hebdomadaires: le Centre des sarcomes du CHUV compte deux colloques hebdomadaires, un pour les enfants et un pour les adultes. Les cas sont annoncés aux secrétaires qui diffusent ensuite une liste aux participants. Cela permet d'améliorer la préparation à la discussion. Les médecins externes au CHUV sont invités à présenter leurs cas aux colloques pour un deuxième avis.

Les médecins de famille adressent en général le patient directement aux consultants du Centre de sarcomes et se déplacent rarement. Parfois, de spécialistes, tels des chirurgiens externes à l'hôpital, viennent présenter personnellement le cas. Nous constatons actuellement une augmentation du nombre de demandes envoyées par e-mail. Ces cas sont référés à un spécialiste du centre qui présente le cas au nom du médecin référent.

- Documenter les discussions et décisions dans le dossier électronique du patient: un formulaire a été élaboré à cet effet, permettant à tout médecin de l'hôpital d'accéder rapidement ces informations en cas de besoin. Au besoin, une lettre peut être géné- rée à partir de ce formulaire et adressée au médecin traitant ou spécialiste qui a référé le cas.

Comme la formation est un objectif prioritaire des hôpitaux universitaires, les assistants et chefs de clinique sont encouragés à participer aux colloques hebdomadaire et à y présenter leurs cas [15]. Les médecins-cadre sont en charge de leur supervision.

\section{Adaptation des recommandations internationales de pratique clinique}

Les recommandations de pratique clinique sont un outil essentiel de la pratique médicale. Elles constituent des documents de référence pour le diagnostic et le traitement de patients [16-18]. Dans le cadre du projet de Centre des sarcomes, des recommandations de prise en charge clinique d'enfants et d'adultes ont été adaptées à partir des recommandations internationales [19]. Disponibles depuis mai 2015, elles sont mises à jour régulièrement. Les médecins spécialistes du Centre des sarcomes du CHUV ont également participé activement à la rédaction de recommandations nationales pour la prise en charge des sarcomes dans le cadre des travaux du Swiss Sarcoma Advisory Board.

\section{Elaboration d'itinéraires cliniques}

Les itinéraires cliniques sont un plan de soin formalisé pour refléter la prise en charge d'une pathologie [20] Ils sont basés sur les recommandations de pratique clinique et tiennent compte des pratiques locales. Leur but est d'améliorer la qualité et la sécurité des soins. Les itinéraires cliniques permettent également de formaliser les interventions de l'équipe de soins de support oncologiques (psychologues, assistant-e-s sociaux/sociales, nutritionnistes et infirmières cliniciennes spécialisées).

Des itinéraires cliniques de diagostic et traitement des sarcomes ont été élaborés au CHUV pour décrire la prise en charge d'adultes et d'enfants, ainsi que la transition de l'une à l'autre conformément aux recommandations nationales en matière de prise en charge des maladies rares (http://www.orphanet.ch/).

\section{Création d'une base de données de centre}

La récolte prospective de données est un outil essentiel dans la pratique médicale contemporaine: elle permet d'améliorer la recherche, l'inclusion de patients dans les essais cliniques, le monitorage de la qualité et la comparaison interhospitalière $[15,16,18]$.

La base de données du Centre des sarcomes du CHUV a été inaugurée le $1^{\mathrm{er}}$ janvier 2015. Cette base regroupe des variables communes à tous les centres et des données spécifiques à la prise en charge des sarcomes. Les données sont collectées dans le respect des cadres 
éthiques et légaux suisses. Elles sont mises ensuite à la disposition de tout médecin souhaitant réaliser une recherche ou publier un article scientifique. La base de données du centre alimente également l'Etude de cohorte suisse des sarcomes, sous la responsabilité du Swiss Sarcoma Advisory Board.

\section{Encourager les médecins de famille et les spécialistes à référer rapidement leurs patients}

Le deuxième objectif du centre était de rendre le centre plus facile d'accès. Le but est d'encourager les médecins de famille et les spécialistes à référer les patients chez qui, soit un sarcome a été diagnostiqué, soit un sarcome est suspecté [21, 22] et éviter ainsi des prises en charge inappropriées.

Pour ce faire, dès 2014 nous avons créé des coordonnées de contact uniques pour les filières pédiatrique et adulte. Celles-ci ont été diffusées au sein des réseaux professionnels et, dès septembre 2016, intégrées au site web du centre (www.chuv.ch/sarcomes):

- Filière pédiatrique: +41213141861,

dmcp.ped.onco[at]chuv.ch

- Filière adulte: +41 2131401 60,

cco.secretariat.sarcomes[at]chuv.ch

Tout médecin peut contacter le centre et être mis en contact avec un chirurgien ou un oncologue spécialisé afin d'obtenir un deuxième avis ou leur référer son patient.

Depuis 2013, un symposium annuel permet également de sensibiliser les professionnels à cette pathologie rare. Le 3 novembre 2016, une conférence publique a également permis de réunir pour la première fois les patients et leurs familles.

\section{Conclusion}

Entre 2009 et 2013, la Ligue suisse contre le cancer a reporté une moyenne de 350 cas annuels de sarcomes. En 2015, le CHUV a pris en charge 80 patients et a examiné un nombre équivalent de patients avec une tumeur bénigne ou d'autres types de lésions. Parmi ceux-ci, 9\% ont subi une biopsie ou chirurgie inappropriée (whoops

\section{L'Equipe interdisciplinaire sarcomes}

\section{du CHUV}

Maja Beck Popovic, MD, Manuel Diezi, MD, et Emma Garcia, MD Service d'hémato-oncologie pédiatrique; Michael Montemurro, MD, Antonia Digklia, MD, MSc, et Laura Jolliet, MSc, Service d'oncologie médicale; Esat Mahmut Ozsahin, MD, Berardino De Bari, MD, et Luis Schiappacasse, MD, Service de radio-oncologie Jean-Marc Joseph, MD, Service de chirurgie pédiatrique; PierreYves Zambelli, MD, et Stéphane Tercier, MD, Service d'orthopédie et traumatologie pédiatrique; Stéphane Cherix, MD, et Hannes Rüdiger, MD, Service d'orthopédie et traumatologie; Nicolas Demartines, MD, et Tobias Zingg, MD, Service de chirurgie viscérale; Michel Gonzalez, MD, Service de chirurgie thoracique; François Saucy, MD, Service de chirurgie vasculaire; Christian Simon, MD, et Martin Broome, MD, Service d'oto-rhino-laryngologie; Thomas Tawadros, MD, Service d'urologie; Leonor Alamo, $M D$, Fabio Becce, MD, Pierre Bize, MD, et Patrick Omoumi, MD, $\mathrm{PhD}$, Service de radiodiagnostic et radiologie interventionnelle; Laurence de Leval, MD, PhD, Igor Letovanec, MD, et Carole Gengler, MD, Service de pathologie clinique

surgeries) au préalable, suggérant que la prévention devrait être améliorée.

Le Centre des sarcomes du CHUV a été officiellement inauguré le $1^{\text {er }}$ octobre 2016 et les indicateurs d'activité suggèrent que, à ce stade, la prise en charge est cohérente avec les objectifs fixés. Comme la prise en charge interdisciplinaire va à l'encontre de l'organisation hospitalière par départements et services, nous sommes conscients qu'un investissement constant est nécessaire pour maintenir les standards fixés.

\section{Remerciements}

Les auteurs souhaitent remercier le personnel administratif et l'équipe de projet du Centre des sarcomes.

\section{Disclosure statement}

Le projet de Centre des sarcomes a été financé par la Direction médicale du CHUV. Actuellement, il est sous la responsabilité financière du Département d'oncologie. Les auteurs ne déclarent aucun conflit d'intérêts en lien avec ce qui précède.

\section{Note}

Le $4^{\mathrm{e}}$ symposium annuel du Centre des sarcomes aura lieu le 16 novembre 2017 de 14 hOO à 18 hoo, au CHUV, auditoire Mathias Mayor (http://www.chuv.ch/sarcomes). 